\title{
Diabetes Mellitus Is a Major Negative Determinant of Coronary Plaque Regression During Statin Therapy in Patients With Acute Coronary Syndrome \\ - Serial Intravascular Ultrasound Observations From the Japan Assessment of Pitavastatin and Atorvastatin in Acute Coronary Syndrome Trial (the JAPAN-ACS Trial) -
}

Takafumi Hiro, MD; Takeshi Kimura, MD*; Takeshi Morimoto, MD**; Katsumi Miyauchi, MD Yoshihisa Nakagawa, MD ${ }^{\ddagger}$; Masakazu Yamagishi, MD*; Yukio Ozaki, MD $\$$; Kazuo Kimura, MD\$; Satoshi Saito, MD ${ }^{\S}$; Tetsu Yamaguchi, MDII; Hiroyuki Daida, $\mathrm{MD}^{\dagger}$; Masunori Matsuzaki, MD ${ }^{\mathbb{I}}$ for the JAPAN-ACS Investigators

Background: The Japan Assessment of Pitavastatin and Atorvastatin in Acute Coronary Syndrome (JAPANACS) trial has found that early aggressive statin therapy in patients with acute coronary syndrome (ACS) significantly reduces the plaque volume (PV) of non-culprit coronary lesions. The purpose of the present study was to evaluate clinical factors that have an impact on plaque regression using statin therapy.

Methods and Results: Serial intravascular ultrasound observations over 8-12 months were performed in 252 ACS patients receiving pitavastatin or atorvastatin. Linear regression analysis identified the presence of diabetes mellitus (DM) and PV at baseline as inhibiting factors, and serum remnant-like particle-cholesterol level at baseline as a significant factor significantly affecting the degree of plaque regression. Significant correlation between \% change of PV and low-density lipoprotein cholesterol (LDL-C) level was found in patients with $D M(n=73, P<0.05$, $r=0.4)$, whereas there was no significant correlation between the 2 parameters in patients without $D M(n=178)$.

Conclusions: The regression of coronary plaque induced by statin therapy after ACS was weaker in diabetic patients than their counterparts. Moreover, vigorous reduction of the LDL-C levels might induce a greater degree of plaque regression in ACS patients with DM. (Circ J 2010; 74: 1165-1174)

Key Words: Acute coronary syndrome; Diabetes mellitus; Intravascular ultrasound; Plaque; Statins

I is now widely accepted that the incidence of secondary cardiovascular events can be significantly reduced by therapy with statins in patients with acute coronary syndrome (ACS). ${ }^{1-3}$ Although the exact pathophysiological mechanism has not yet been clarified, several attempts have been made, including using intravascular imaging modalities, to directly or indirectly evaluate the improvement in the plaque vulnerability with statin therapy. ${ }^{4-7}$

Received October 8, 2009; revised manuscript received February 15, 2010; accepted February 16, 2010; released online May 12 , 2010 Time for primary review: 46 days

Division of Cardiology, Department of Medicine, Nihon University School of Medicine, `Department of Cardiology, Juntendo University School of Medicine, ${ }^{\S}$ Division of Cardiovascular Medicine, Keiai Hospital, "Toranomon Hospital, Tokyo, *Department of Cardiovascular Medicine, **Center for Medical Education, Kyoto University Graduate School of Medicine, Kyoto, † Department of Cardiology, Tenri Hospital, Tenri, \#Division of Cardiovascular Medicine, Kanazawa University Graduate School of Medicine, Kanazawa, 㳯epartment of Cardiology, Fujita Health University, Toyoake, ${ }^{\S}$ Division of Cardiology, Yokohama City University Medical Center, Yokohama and IUIDivision of Cardiology, Department of Medicine and Clinical Science, Yamaguchi University Graduate School of Medicine, Ube, Japan

The Japan Heart Foundation funded this study with an unrestricted grant from Kowa Pharmaceutical. Kowa Pharmaceutical participated in the preparation of the study design, but the investigators or an independent Clinical Research Coordinator (see Acknowledgment) made the final decision on the study design, database maintenance, made manuscript, and submission of the article; an independent statistician (see Appendix 1) analyzed the data.

Trial Registration: ClinicalTrials.gov Identifier: NCT002429; http://clinicaltrials.gov/ct2/show/NCT00242944

Mailing address: Masunori Matsuzaki, MD, Division of Cardiology, Department of Medicine and Clinical Science, Yamaguchi University Graduate School of Medicine, Ube 755-8505, Japan. E-mail: masunori@yamaguchi-u.ac.jp

ISSN-1346-9843 doi:10.1253/circj.CJ-09-0766

All rights are reserved to the Japanese Circulation Society. For permissions, please e-mail: cj@j-circ.or.jp 


\begin{tabular}{|ccc|}
\hline \multicolumn{3}{c}{ Table 1. Factors Influencing Degree of Plaque Regression } \\
Factors & $\beta$ & \multicolumn{1}{c|}{$95 \% \mathbf{C l}$} \\
Univariate analysis & & \\
Diabetes (absent-present) & 6.6 & $2.9-10.4$ \\
Gender (women-men) & 5.1 & $0.68-9.6$ \\
Height at baseline & 0.20 & $-0.01-0.41$ \\
Plaque volume at baseline & 0.053 & $-0.0-0.11$ \\
Vessel volume at baseline & 0.028 & $-0.0-0.058$ \\
RLP-C level at baseline & -0.59 & $-1.3-0.086$ \\
ApoE level at baseline & -1.3 & $-2.8-0.22$ \\
HbA1c at baseline & 2.4 & $0.94-3.8$ \\
Multivariate analysis & & \\
Diabetes (absent-present) & 7.3 & $3.6-10.9$ \\
Plaque volume at baseline & 0.069 & $0.017-0.12$ \\
RLP-C at baseline & -0.70 & -1.4 to -0.048 \\
\hline
\end{tabular}

RLP-C, remnant-like particle-cholesterol; ApoE, apolipoprotein E; $\mathrm{Hb}$, hemoglobin.

\section{Editorial p 1073}

The Japan Assessment of Pitavastatin and Atorvastatin in Acute Coronary Syndrome (JAPAN-ACS) study was performed as a prospective, randomized open-label parallelgroup study with a blind endpoint evaluation at 33 centers, to comparatively examine the effect of 8-12-months' treatment with pitavastatin and atorvastatin on the degree of coronary plaque regression in non-culprit lesions of the culprit vessel treated by PCI in patients with ACS. ${ }^{8}$ The results of this analysis demonstrated the non-inferiority of pitavastatin $4 \mathrm{mg} /$ day to atorvastatin $20 \mathrm{mg} /$ day, with an approximately $17 \%$ regression of the plaque volume (PV), suggesting that the effect of inducing plaque regression can be generalized to other statins. The degree of this effect varied widely among patients, regardless of the baseline low-density lipoprotein cholesterol (LDL-C) level, and it would be important to clarify its critical determinants. Previous studies have documented several determinants of the degree of plaque progression/regression in patients with stable coronary artery disease, such as gender, ${ }^{9}$ blood pressure, ${ }^{10}$ obesity, ${ }^{11}$ and use/non-use of $\beta$-blockers. ${ }^{12}$ The major baseline determinants of the degree of plaque regression have not, however, yet been determined in patients with ACS.

The purpose of the present sub-analysis of the JAPANACS study was therefore to identify the major baseline determinants of the degree of plaque regression in ACS patients under treatment with statins, on multivariate analysis. The baseline parameters examined included the clinical patient characteristics, serum lipid profile, concomitantly administered drugs, intravascular ultrasound (IVUS) parameters, and presence/absence of underlying diseases, such as diabetes mellitus (DM). This study was performed in the entire patient population, using the full analysis set, of the JAPAN-ACS study, because the regressive effect of the 2 statins was shown to be equivalent in that study. ${ }^{8}$

\section{Methods}

\section{Study Subjects and Ethics Considerations}

The present study was a sub-analysis of the JAPAN-ACS study. ${ }^{8}$ Description of the present study design is published elsewhere. ${ }^{13}$ In brief, ACS patients selected were $>20$ years of age with hypercholesterolemia and had undergone successful PCI under IVUS guidance. They were found to have coronary plaques $(>500 \mu \mathrm{m}$ in thickness, or percent plaque area $\geq 20 \%$ ) in the culprit vessel at least $5 \mathrm{~mm}$ away from the PCI-treated lesions. ACS was defined as unstable angina pectoris, non-ST-elevation myocardial infarction (MI) or STelevation MI. The diagnosis of ACS was made based on the fulfillment of at least 2 of the following three criteria: (1) evidence of coronary ischemia on ECG; (2) increase ( $\geq 2$-fold) in the serum creatinine kinase (CK) or CK-MB levels and/or troponin-T positivity; and (3) presence of symptoms suggestive of ACS. DM and other complications were diagnosed by the attending physicians.

The study was conducted in conformity with the principles of the Declaration of Helsinki, and with the approval of the institutional review boards of all of the 33 participating institutions. Written informed consent for participation was obtained from all of the patients enrolled.

\section{IVUS Procedure and Examination}

Details of the IVUS procedure and examination are documented elsewhere. ${ }^{8}$ In brief, following IVUS-guided PCI for the culprit lesion in the patients with ACS, a final IVUS examination for analysis was performed in the culprit vessel. The IVUS catheter Atlantis SR Pro2 (Boston Scientific, Natick, MA, USA) was used, and a motorized pullback device withdrew the transducer at the speed of $0.5 \mathrm{~mm} / \mathrm{s}$. The consoles used were the ClearView or Galaxy 2 system (Boston Scientific). The same imaging system with the same type of IVUS catheter was used for both the baseline and the follow-up examinations.

Two independent experienced investigators performed the quantitative IVUS analysis at the central core-laboratory. The target segment for analysis was identified at a non-PCI site of the culprit vessel ( $>5 \mathrm{~mm}$ proximal or distal to the PCI site) based on some reproducible indices. Manual tracing was performed in every $0.1-\mathrm{mm}$ cross-section and the software (echoPlaque2, INDEC systems, Santa Clara, USA) automatically interpolated the tracings of 5 cross-sections between 2 manually traced images. Therefore, the volume was calculated from each of the $0.017-\mathrm{mm}$-apart segments.

\section{IVUS Parameters}

The primary endpoint of the JAPAN-ACS study was the percent change in coronary PV, which was calculated as follows:

$$
\frac{\text { PV (follow up) }-\mathrm{PV}(\text { baseline })}{\mathrm{PV}(\text { baseline })} \times 100
$$

Coronary PV was calculated using the sum of the differences between the external elastic membrane (EEM) cross-sectional area (CSA) and the lumen CSA across all evaluated frames as: $\mathrm{PV}=\sum$ (EEMcsA-LUMENcsA $) \times($ slice thickness $)$.

The major secondary endpoints were the nominal change of the percent PV (\%PV) and nominal change of the normalized PV (NPV; follow-up PV minus baseline PV, respectively). The \%PV was calculated using the following formula:

$$
\% \mathrm{PV}=\frac{\sum\left(\text { EEMCSA }_{\text {LUMEN }}{ }_{\text {CSA }}\right)}{\sum(\text { EEMCSA })} \times 100
$$

NPV was calculated as:

$$
\mathrm{NPV}=\mathrm{PV} \times \frac{\text { LMED }}{\text { LMEASURED }}
$$

where $\mathrm{LMED}=$ the median value of observed length in all subjects and LMEAURED= observed length for each plaque. 


\begin{tabular}{|c|c|c|c|}
\hline Characteristic & $\begin{array}{c}\text { Diabetes } \\
(n=74)\end{array}$ & $\begin{array}{c}\text { Non-diabetes } \\
\quad(n=178)\end{array}$ & $\begin{array}{l}\mathrm{P} \text { between } \\
\text { groups }\end{array}$ \\
\hline Age (years) & $62.8 \pm 10.5$ & $62.3 \pm 11.3$ & 0.7 \\
\hline Male & $61(82.4)$ & $145(81.5)$ & 0.9 \\
\hline BMI $\left(\mathrm{kg} / \mathrm{m}^{2}\right)$ & $24.9 \pm 3.7$ & $24.1 \pm 3.4$ & 0.1 \\
\hline Waist circumference $(\mathbf{c m})$ & $88.7 \pm 8.1$ & $86.5 \pm 9.4$ & 0.08 \\
\hline Hypertension & $52(70.3)$ & $105(59.0)$ & 0.09 \\
\hline Family history of CAD & $13(17.6)$ & $32(18.0)$ & 0.9 \\
\hline Smoking & $36(48.7)$ & $83(46.6)$ & 0.8 \\
\hline Alcohol drinker & $40(54.1)$ & $81(45.5)$ & 0.2 \\
\hline Type of ACS & & & 0.4 \\
\hline STEMI & $45(60.8)$ & $117(65.7)$ & \\
\hline NSTEMI & $14(18.9)$ & $22(12.4)$ & \\
\hline UAP & $15(20.3)$ & $39(21.9)$ & \\
\hline Abnormal $Q$ wave & $25(33.8)$ & $61(34.2)$ & 0.9 \\
\hline Max CK median (IQR) (IU/L) & $1,228(289-2,663)$ & $1,271(224-2,868)$ & 0.98 \\
\hline Culprit vessel & & & 0.08 \\
\hline RCA & $27(36.5)$ & $54(30.3)$ & \\
\hline LAD & $32(43.2)$ & $104(58.4)$ & \\
\hline LCx & $15(20.3)$ & $19(10.7)$ & \\
\hline LMT & 0 & $1(0.6)$ & \\
\hline Analysis segment & & & 0.01 \\
\hline Proximal to the treated site & $60(81.1)$ & $116(65.2)$ & \\
\hline Distal to the treated site & $14(18.9)$ & $62(34.8)$ & \\
\hline Type of stent & & & 0.97 \\
\hline BMS & $49(66.2)$ & $117(65.7)$ & \\
\hline DES & $23(31.1)$ & $57(32.0)$ & \\
\hline Other than stent (POBA) & $2(2.7)$ & $4(2.3)$ & \\
\hline \multicolumn{4}{|l|}{ Concomitant drugs } \\
\hline Aspirin & $74(100)$ & $174(97.8)$ & 0.2 \\
\hline Ticlopidine & $63(85.1)$ & $146(82.0)$ & 0.5 \\
\hline Clopidogrel & $4(5.4)$ & $13(7.3)$ & 0.6 \\
\hline$\beta$-blocker & $36(48.7)$ & $80(44.9)$ & 0.6 \\
\hline ACE inhibitor & $21(28.4)$ & $53(29.8)$ & 0.8 \\
\hline ARB & $41(55.4)$ & $84(47.2)$ & 0.2 \\
\hline Insulin & $10(13.5)$ & 0 & - \\
\hline PPAR- $\gamma$ agonist & $10(13.5)$ & 0 & - \\
\hline Sulfonyl urea & $20(27.0)$ & 0 & - \\
\hline$\alpha-\mathrm{Gl}$ & $21(28.3)$ & 0 & - \\
\hline Calcium blocker & $17(23.0)$ & $32(18.0)$ & 0.4 \\
\hline Nitrate & $13(17.6)$ & $25(14.0)$ & 0.5 \\
\hline Diuretic & $9(12.2)$ & $10(5.6)$ & 0.07 \\
\hline Aldosterone blocker & $2(2.7)$ & $3(1.7)$ & 0.6 \\
\hline Digitalis & $4(5.4)$ & $1(0.6)$ & 0.03 \\
\hline Other anti platelet agents & $4(5.4)$ & $13(7.3)$ & 0.8 \\
\hline Warfarin & $4(5.4)$ & $3(1.7)$ & 0.2 \\
\hline Anti arrhythmic agent & 0 & $2(1.1)$ & 1.0 \\
\hline
\end{tabular}

Data are expressed as numbers (percentage) unless otherwise specified.

Continuous variables are represented by mean $\pm S D$ or median (IQR).

$\mathrm{BMI}$, body mass index; CAD, coronary artery disease; ACS, acute coronary syndrome; STEMI, ST-elevation myocardial infarction; NSTEMI, non-STEMI; UAP, unstable angina pectoris; CK, creatinine kinase; IQR, intra quartile range; $R C A$, right coronary artery; LAD, left anterior descending artery; LCx, left circumflex branch; LMT, left main trunk; BMS, bare metal stent; DES, drug-eluting stent; POBA, plain old balloon angioplasty; ACE, angiotensin-converting enzyme; ARB, angiotensin receptor blocker; $\alpha$-Gl, $\alpha$-glucosidase inhibitor.

\section{Statistical Analysis}

We used the full analysis set (FAS) of the JAPAN-ACS study for this sub-analysis. Patients were included in the FAS if they had ACS and measurable IVUS lesions both at enroll- ment and at follow up. To identify the factors that might influence the $\%$ change of the coronary PV, we first defined 83 variables at baseline as potential factors (Appendix 2). We developed a univariate and multivariate general linear model 


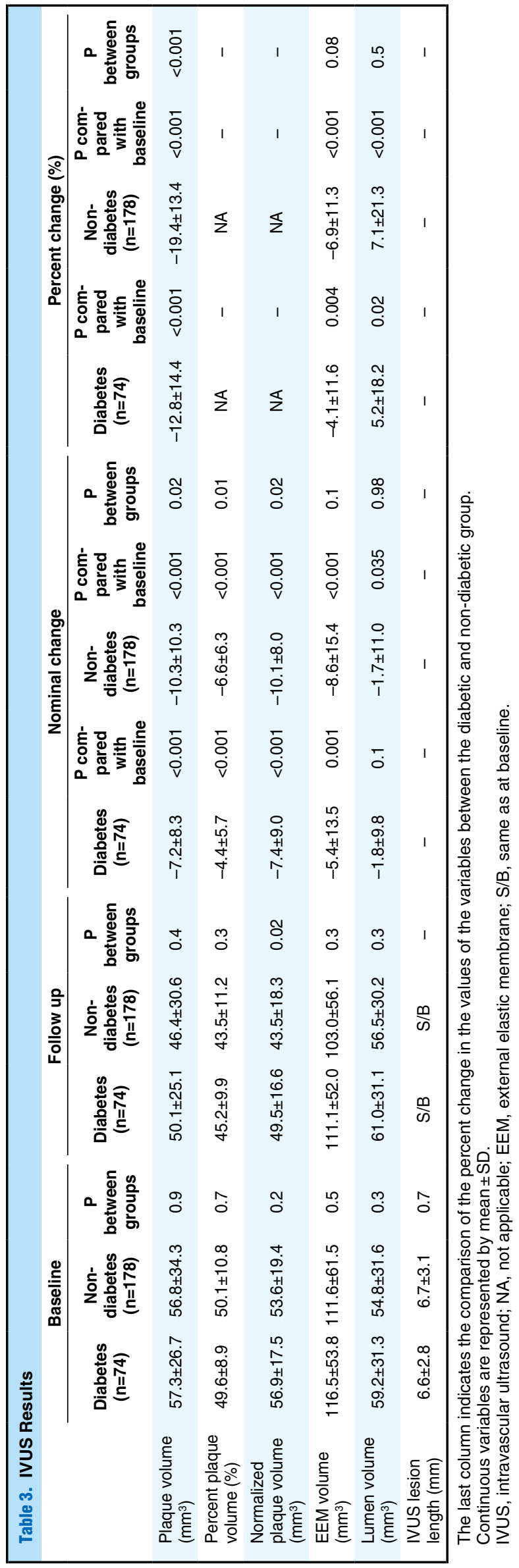

to assess the relationship between the $\%$ change of the coronary PV and each potentially significant variable. Variables that met the following criteria were included for the univariate analysis conducted to determine the association with the primary endpoint: (1) measurements obtained in $\geq 50 \%$ of the total population; and (2) frequency $\geq 10 \%$ of the total population for each nominal or ordinal variable. The variables showing correlation with the $\%$ change in $\mathrm{PV}$ with $\mathrm{P}<0.1$ were included in the multivariate analyses. We finally developed a multivariable model using the variables selected on univariate analysis, followed by backward model selection with $\mathrm{P}<0.05$.

Based on the results of the multivariable model, we identified DM as a strong determinant of the coronary plaque regression. We thus divided the total subjects into 2 groups according to the diabetes status. Following the descriptive statistics, comparisons of continuous variables between 2 groups were performed using a two-sample t-test or Wilcoxon's rank-sum test, and those between the parameters at baseline and follow up were performed using one-sample t-tests or Wilcoxon's signed rank test according to their distributions. Comparisons of categorical variables between 2 groups were performed using the chi-square and Fisher's exact tests. We used general linear models to assess the relationships between the $\%$ change of the coronary PV and several of the variables examined, including the serum lipid profile at the 8-12-month follow up. The significance level was set at $5 \%$ for the twosided test (and $2.5 \%$ for one-sided test).

All the statistical analyses were performed using the SAS System Release 9.1 (SAS institute, Cary, NC, USA).

\section{Results}

\section{Multivariate Analysis}

According to the selection criteria, the presence of DM, gender and six baseline parameters, including the height, PV, vessel volume, remnant-like particle-cholesterol (RLP-C) level, apolipoprotein (Apo) E level and hemoglobin $\mathrm{A}_{1 \mathrm{c}}\left(\mathrm{HbA}_{1 \mathrm{c}}\right.$ ), were selected on univariate analysis as factors determining the degree of plaque regression (Table 1). The multivariate analysis identified the presence of DM, PV at baseline and RLP-C level at baseline as the factors that were significantly associated with the degree of plaque regression. In this analysis, a positive estimate corresponded to a lower likelihood of regression. The estimate for the presence of DM was $7.3(95 \%$ confidence interval (CI): 3.6-10.9), for the PV at baseline it was 0.069 (95\%CI: $0.017-0.12$ ), and for the RLP-C level at baseline it was -0.70 (95\% CI: -1.4 to -0.048$)$. Based on the multivariate model, we identified the presence/absence of DM as being strongly correlated with the primary endpoint. We therefore conducted further analyses after subdividing the participants into a diabetic and non-diabetic group.

\section{Patient Characteristics}

The 252 patients (125 from the pitavastatin group and 127 from the atorvastatin group) were divided into those with DM (diabetic group; $n=74$ ) and those without DM (non-diabetic group; $n=178$ ). All the baseline demographic characteristics were similar between the 2 groups, except for the location of the plaque segment of interest (Table 2). Segments proximal to the PCI site for IVUS evaluation were included more frequently in the diabetic group than in the non-diabetic group (81.1\% vs $65.2 \%, \mathrm{P}=0.012)$. In the diabetic group, $13.5 \%$ of the patients were receiving insulin, $13.5 \%$ were under treatment with a PPAR- $\gamma$ agonist, $27 \%$ were under treatment with 


\section{$\%$ change in plaque volume}

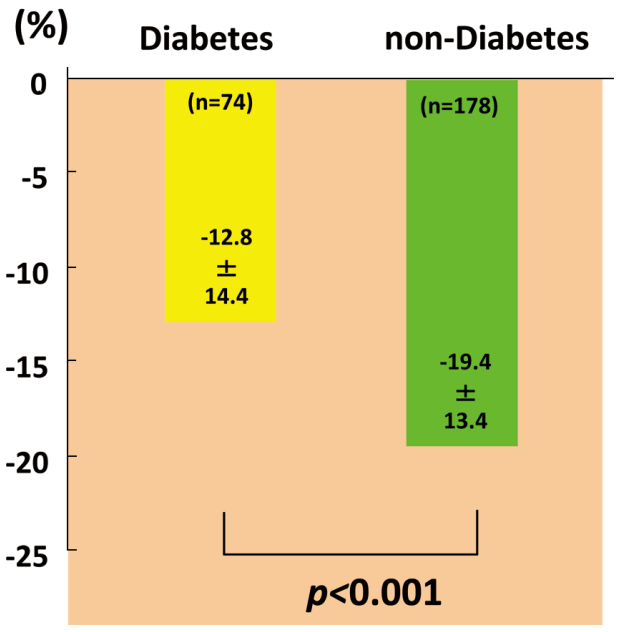

\% change in LDL-C

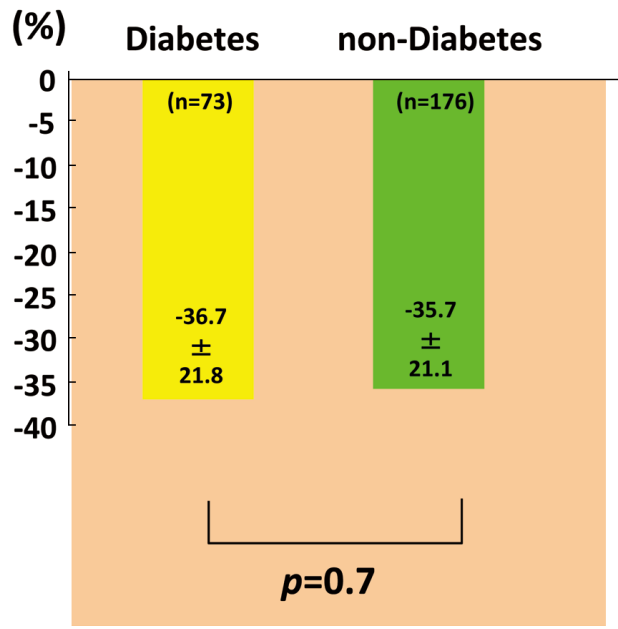

Figure 1. Plaque volume and low-density lipoprotein cholesterol (LDL-C) change in patients with or without diabetes mellitus. The percent change in plaque volume at the 8-12-month follow up was significantly lower in the diabetic group as compared with that in the non-diabetic group, although the percent change in LDL-C level during the observation period was not significantly different between the 2 groups. Values are mean $\pm S D$.

\begin{tabular}{|c|c|c|c|c|c|c|c|}
\hline & \multicolumn{2}{|c|}{ Baseline } & \multicolumn{2}{|c|}{ Follow up } & \multicolumn{3}{|c|}{ Percent change (\%) } \\
\hline & $\begin{array}{c}\text { Diabetes } \\
(n=74)\end{array}$ & $\begin{array}{c}\text { Non-diabetes } \\
(n=178)\end{array}$ & $\begin{array}{c}\text { Diabetes } \\
(n=74)\end{array}$ & $\begin{array}{c}\text { Non-diabetes } \\
(n=178)\end{array}$ & $\begin{array}{c}\text { Diabetes } \\
(n=74)\end{array}$ & $\begin{array}{c}\text { Non-diabetes } \\
(n=178)\end{array}$ & $\begin{array}{c}P \text { between } \\
\text { groups }\end{array}$ \\
\hline $\mathrm{TC}(\mathrm{mg} / \mathrm{dl})$ & $197.6 \pm 35.7$ & $197.0 \pm 36.1$ & $153.0 \pm 34.0$ & $151.7 \pm 29.2$ & $-21.8 \pm 16.0$ & $-21.7 \pm 17.2$ & 0.97 \\
\hline LDL-C (mg/dl) & $131.5 \pm 31.9$ & $132.7 \pm 32.5$ & $81.5 \pm 28.1$ & $83.0 \pm 24.4$ & $-36.7 \pm 21.8$ & $-35.7 \pm 21.1$ & 0.7 \\
\hline $\mathrm{TG}(\mathrm{mg} / \mathrm{dl})$ & $126.1 \pm 55.8$ & $114.6 \pm 55.3$ & $124.1 \pm 61.6$ & $123.6 \pm 73.8$ & $13.5 \pm 71.6$ & $20.9 \pm 66.6$ & 0.4 \\
\hline $\mathrm{HDL}-\mathrm{C}(\mathrm{mg} / \mathrm{dl})$ & $45.0 \pm 10.8$ & $43.9 \pm 9.4$ & $49.0 \pm 13.4$ & $47.4 \pm 11.7$ & $11.6 \pm 22.9$ & $7.8 \pm 22.2$ & 0.2 \\
\hline RLP-C (mg/dl) & $4.4 \pm 2.4$ & $4.3 \pm 2.6$ & $3.9 \pm 2.5$ & $3.9 \pm 3.3$ & $1.6 \pm 70.9$ & $7.0 \pm 89.4$ & 0.7 \\
\hline Small dense LDL (RM) & $0.35 \pm 0.04$ & $0.36 \pm 0.05$ & $0.34 \pm 0.04$ & $0.34 \pm 0.03$ & $-1.8 \pm 11.9$ & $-3.4 \pm 11.3$ & 0.3 \\
\hline Non-HDL-C (mg/dl) & $152.1 \pm 32.4$ & $152.4 \pm 33.8$ & $104.0 \pm 34.0$ & $104.2 \pm 26.7$ & $-30.8 \pm 21.2$ & $-30.1 \pm 19.3$ & 0.8 \\
\hline LDL-C/HDL-C & $3.1 \pm 0.9$ & $3.1 \pm 0.9$ & $1.8 \pm 0.8$ & $1.8 \pm 0.6$ & $-40.4 \pm 26.5$ & $-39.2 \pm 18.9$ & 0.7 \\
\hline hs-CRP median:IQR (mg/L) & $\begin{array}{c}16.9 \\
(5.3-42.0)\end{array}$ & $\begin{array}{c}20.1 \\
(5.4-68.0)\end{array}$ & $\begin{array}{c}0.54 \\
(0.26-1.5)\end{array}$ & $\begin{array}{c}0.54 \\
(0.32-1.0)\end{array}$ & $\begin{array}{c}-95.7 \\
(-99.1 \text { to }-83.5)\end{array}$ & $\begin{array}{c}-96.9 \\
(-99.1 \text { to }-89.4)\end{array}$ & $0.3^{\star}$ \\
\hline WBC:IQR (cells/ $\mu \mathrm{l})$ & $\begin{array}{c}9,450 \\
(7,675-11,250)\end{array}$ & $\begin{array}{c}8,900 \\
(7,175-11,225)\end{array}$ & $\begin{array}{c}6,400 \\
(5,050-7,600)\end{array}$ & $\begin{array}{c}5,905 \\
(5,145-7,000)\end{array}$ & $\begin{array}{c}-33.3 \\
(-48.8 \text { to }-12.0)\end{array}$ & $\begin{array}{c}-32.7 \\
(-44.0 \text { to }-19.1)\end{array}$ & $0.8^{*}$ \\
\hline $\mathrm{HbA}_{1 \mathrm{c}}(\%)$ & $7.3 \pm 1.4$ & $5.4 \pm 0.4$ & $6.8 \pm 1.4$ & $5.6 \pm 0.4$ & $-0.44 \pm 1.42^{\dagger}$ & $0.14 \pm 0.30^{\dagger}$ & $<0.001$ \\
\hline
\end{tabular}

Continuous variables are represented by mean \pm SD or median (IQR).

*Wilcoxon sign rank test. †Nominal change.

HDL-C, high density lipoprotein-cholesterol; LDL-C, low density lipoprotein-cholesterol; hs-CRP, high-sensitivity C-reactive protein; WBC, white blood cells. Other abbreviations see in Tables 1,2 .

SI conversions: to convert total cholesterol, LDL-C, HDL-C, RLP-C, non-HDL-C to mmol/L, multiply by 0.0259 ; PTX3 to $\mu \mathrm{g} / \mathrm{L}$, multiply values by 1 .

a sulfonyl urea, and $28.3 \%$ were under treatment with an $\alpha$ glucosidase inhibitor.

\section{IVUS Parameters}

There were no significant differences in the baseline IVUS parameters between the diabetic and non-diabetic patients (Table 3). The percent change in PV at the 8-12-month follow up, however, was significantly lower in the diabetic group as compared with that in the non-diabetic group $(-12.8 \pm$ $14.4 \%$ vs $-19.4 \pm 13.4 \%, \mathrm{P}<0.001$ ) (Figure 1). The nominal change in the \%PV or NPV were also significantly decreased in the diabetic group as compared with that in the non-dia- betic group $(\mathrm{P}=0.01$ and $\mathrm{P}=0.02$, respectively). In contrast, no significant difference in the percent change of the EEM or lumen volume was observed between the 2 groups $(\mathrm{P}=0.08$ and $\mathrm{P}=0.5$, respectively; Table 3 ). Although segments proximal to the PCI site were measured more frequently in the diabetic group than in the non-diabetic group, the percent change of the PV was similar between the proximal site and distal site. Furthermore, there was no difference in baseline $\% \mathrm{PV}$ between distal and proximal segments in each group.

\section{Laboratory Results}

Significant decrease of the LDL-C level from 131.5 \pm 
Diabetic patients

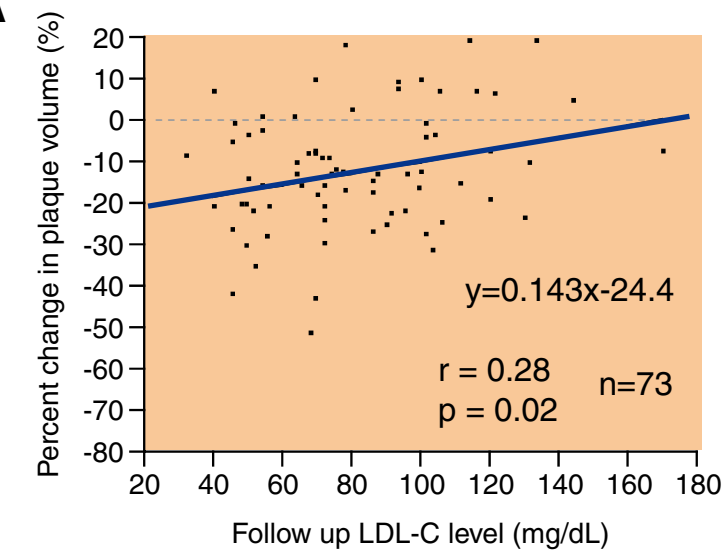

C

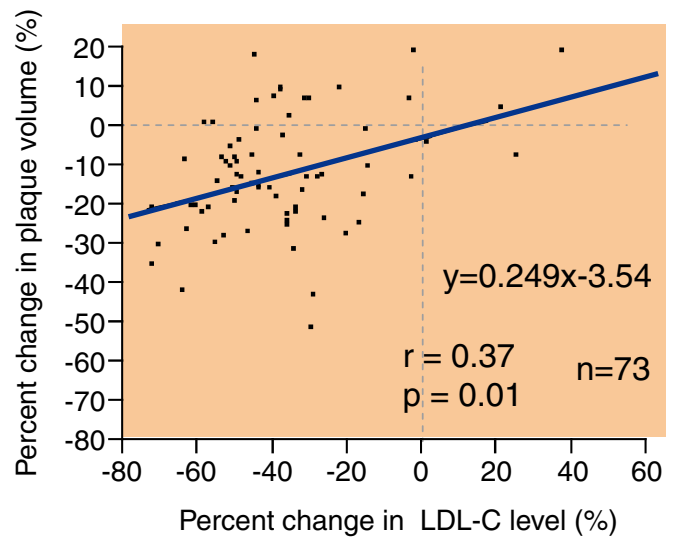

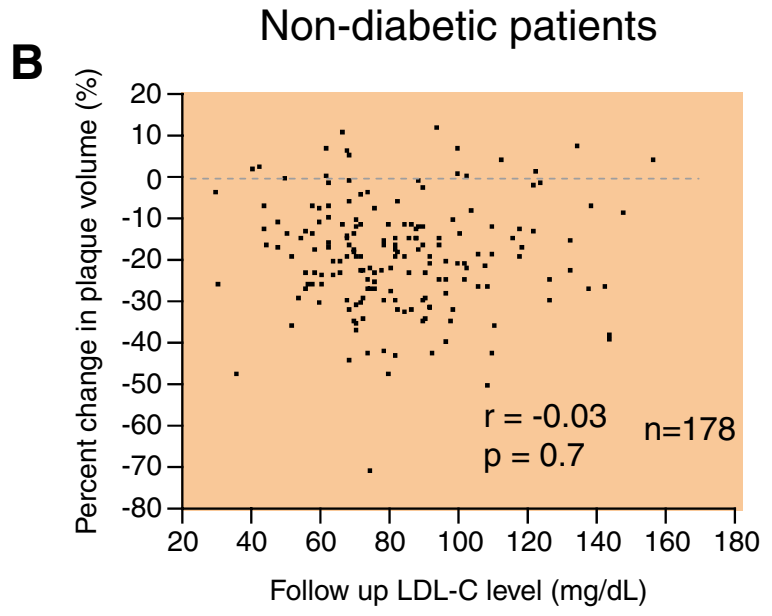

D

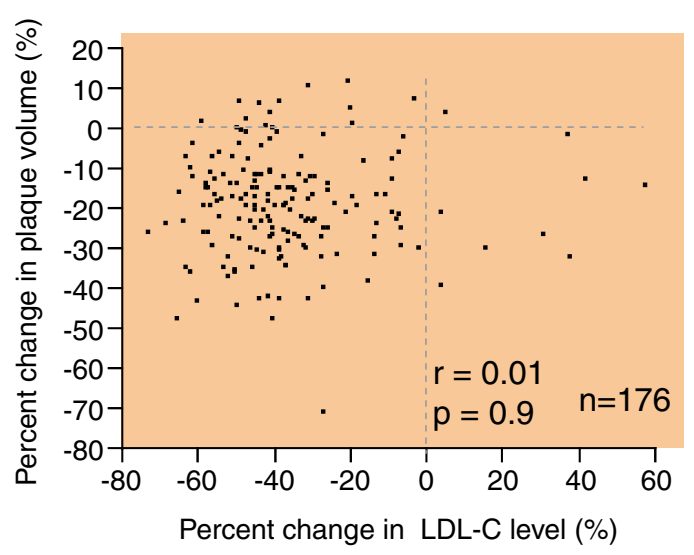

Figure 2. Relationship between the low-density lipoprotein cholesterol (LDL-C) level and percent change in plaque volume in patients with and without diabetes. (A, C) There were significant correlations between the follow-up LDL-C level, as well as the percent change of the LDL-C level, and the percent change in plaque volume during the 8-12-month follow up in patients with diabetes. (B, D) In contrast, no such relationships were detected in the non-diabetic patients.

$31.9 \mathrm{mg} / \mathrm{dl}(3.40 \pm 0.83 \mathrm{mmol} / \mathrm{L})$ at baseline to $81.5 \pm 28.1 \mathrm{mg} / \mathrm{dl}$ $(2.11 \pm 0.73 \mathrm{mmol} / \mathrm{L})$ at the $8-12$-month follow up $(\mathrm{P}<0.001$ vs baseline) was observed in the diabetic group, and from $132.7 \pm 32.5 \mathrm{mg} / \mathrm{dl}(3.44 \pm 0.84 \mathrm{mmol} / \mathrm{L})$ at baseline to $83.0 \pm$ $24.4 \mathrm{mg} / \mathrm{dl}(2.14 \pm 0.63 \mathrm{mmol} / \mathrm{L} ; \mathrm{P}<0.001$ vs baseline $)$ at the 8-12-month follow up in the non-diabetic group (Table 4). The LDL-C levels at the baseline and at the 8-12-month follow up and its percent change during the observation period were similar between the 2 groups (Figure 1). The high-density lipoprotein cholesterol (HDL-C) level also had a comparable increase in the 2 groups. The serum levels of inflammatory markers, namely, hs-CRP and PTX3, and the white blood cell count, were elevated to equivalent degrees at baseline, and no significant differences in the percent changes of the parameters were observed between the 2 groups. The $\mathrm{HbA}_{1 c}$ level at baseline was significantly higher in the diabetic group as compared with that in the non-diabetic group (7.3 $\pm 1.4 \%$ vs $5.4 \pm 0.4 \%, \mathrm{P}<0.001)$. During the follow-up period, the $\mathrm{HbA}_{1 \mathrm{c}}$ level decreased significantly in the diabetic group (from $7.3 \pm 1.4 \%$ to $6.8 \pm 1.4 \%, \mathrm{P}=0.012$ ), but increased significantly, although only slightly, in the non-diabetic group (from $5.4 \pm 0.4 \%$ to $5.6 \pm 0.4 \%, \mathrm{P}<0.001$ ).

\section{Degree of Plaque Regression and Biomarkers}

Significant correlation was observed between the LDL-C level at the 8-12-month follow up and the percent change of the PV in the diabetic group $(\mathrm{r}=0.28, \mathrm{P}=0.017)$, whereas no such significant correlation was observed in the non-diabetic group ( $\mathrm{r}=-0.03, \mathrm{P}=0.7$; Figure 2 ). In addition, the percent change of the LDL-C level during the study period was also significantly correlated with the percent change of the PV $(\mathrm{r}=0.37, \mathrm{P}=0.011)$ in the diabetic group, but not in the nondiabetic patients $(r=0.01, P=0.9)$. Furthermore, significant and close correlations between the LDL-C level at the 812-month follow up as well as the percent change of this parameter during the study period with the percent change of the PV were observed in diabetic patients with baseline $\mathrm{HbA} 1 \mathrm{c}$ levels higher than or equal to the median, that is, $7.0 \%(\mathrm{r}=0.49$, $\mathrm{P}=0.0027$, and $\mathrm{r}=0.45, \mathrm{P}=0.0056$, respectively), whereas no significant correlation among these parameters were observed in the diabetic patients with baseline $\mathrm{HbA}_{1}$ levels $<7.0 \%$ $(r=0.06, P=0.7$, and $r=0.24, P=0.2$, respectively; Figure 3$)$.

There were also statistically significant correlations between the percent changes of other lipid parameters such as the total cholesterol (TC; $r=0.37, P=0.0012)$, RLP-C ( $r=0.25$, $\mathrm{P}=0.032)$, non-HDL-C $(\mathrm{r}=0.37, \mathrm{P}=0.017)$ and ApoB $(\mathrm{r}=0.33$, 
A

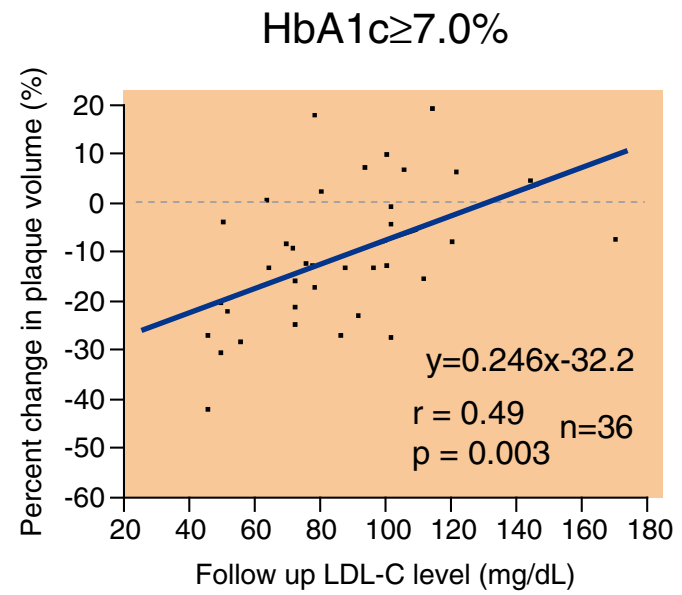

C

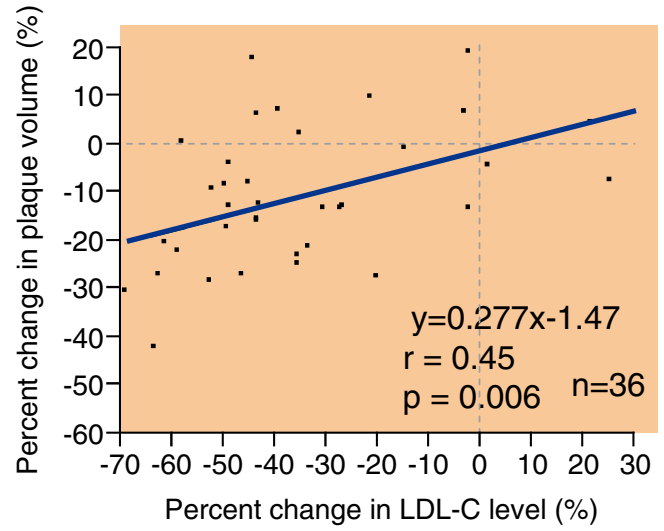

B

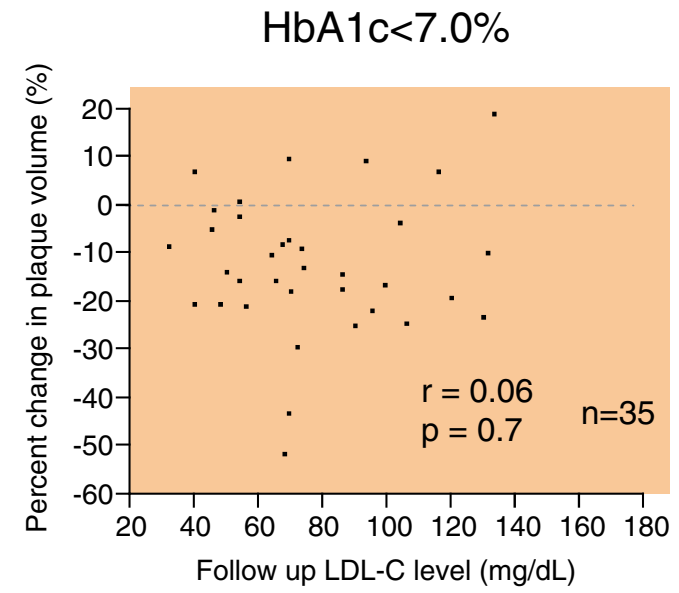

D

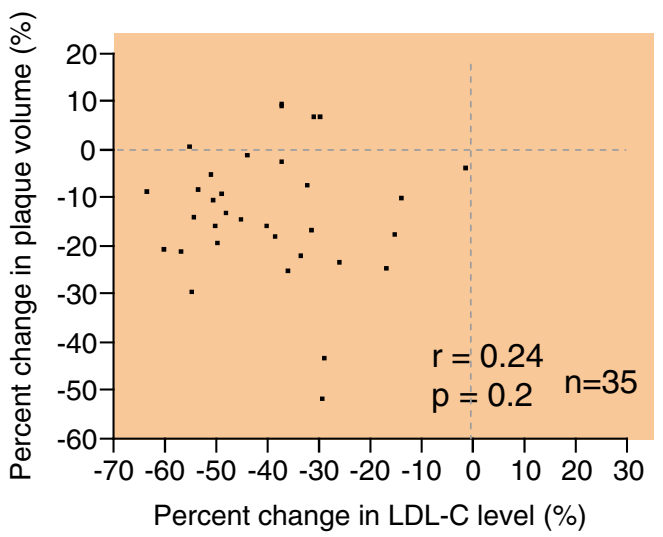

Figure 3. Relationships between the low-density lipoprotein cholesterol (LDL-C) levels and the percent change in plaque volume in patients with diabetes (classified into subgroups by the baseline hemoglobin (Hb)A 1 c level). (A, C) There were significant correlations between the follow-up LDL-C level, as well as the percent change of the LDL-C level, and the percent change in plaque volume during the 8-12-month follow up, especially in the diabetic patients with baseline $\mathrm{HbA}_{1 c}$ levels $\geq 7.0 \%$. (B, D) No such significant relationships, however, were detected in the diabetic patients with baseline $\mathrm{HbA}_{1 \mathrm{c}}$ levels $<7.0 \%$.

$\mathrm{P}=0.0055)$ and the percent change of the PV in the diabetic group, while no such correlations were observed in the nondiabetic group.

\section{Major Adverse Cardiovascular Events (MACE) and Safety Profile}

There was a significant difference in the prevalence of MACE between the diabetic and non-diabetic groups $(29.9 \%$ vs $18.2 \%, \mathrm{P}=0.026)$. Among the MACE components, target lesion revascularization (TLR) tended to be observed at a higher frequency in the diabetic group than in the non-diabetic group (17.2\% vs $9.6 \%, \mathrm{P}=0.06)$. In regard to the other components, the incidences of MI (1.2\% vs $1.0 \%, \mathrm{P}>0.99)$, target vessel revascularization (non-TLR; $6.9 \%$ vs $5.3 \%$, $\mathrm{P}=0.6)$ and revascularization of the non-culprit vessels $(6.9 \%$ vs $5.3 \%, \mathrm{P}=0.6$ ) were similar between the diabetic and nondiabetic groups.

There were no significant differences in the prevalence of adverse events between the diabetic group and the nondiabetic group.

\section{Discussion}

This sub-analysis of the multi-center JAPAN-ACS study using multivariate analysis showed that the presence/absence of DM, the PV and the RLP-C level at baseline were independently associated with the degree of plaque regression induced by statin therapy in patients with ACS. In particular, the percent change in PV during the 8-12-month follow-up period was significantly attenuated in the diabetic group as compared with that in the non-diabetic group. This result was consistent with that reported from a previous clinical outcome study, in which DM patients had a worse prognosis despite advanced treatment for ACS as compared to nonDM patients. ${ }^{14}$ There were significant correlations, however, between the percent change in PV and percent change of the LDL-C level or follow-up LDL-C level in patients with DM, while no such significant correlations were observed in the patients without DM. These data indicated that the presence of DM was one of the major deterrents of plaque regression induced by statin therapy in patients with ACS. In addition, the results also suggested that more vigorous reduction of the LDL-C levels might induce a greater degree of regression of the PV in ACS patients with DM. 
Because the baseline patient profiles were similar between the diabetic and non-diabetic patients with ACS, the differences between the 2 groups in terms of the degree of plaque regression or the relation of this parameter with the changes in the LDL-C levels during statin therapy might be attributable to differences in the baseline characteristics of plaque. This may be supported by the present results, in which a significant difference in the prevalence of MACE was found between the diabetic and non-diabetic groups. Nicholls et al documented, based on a pooled analysis of 5 IVUS trials conducted on a total of 2,237 patients, that diabetic patients had a greater percent or total atheroma volume, with more rapid progression of the $\mathrm{PV}$ and inadequate compensatory remodeling. ${ }^{15}$ This suggested the existence of a unique pathogenetic mechanism for plaque formation in diabetic patients, although there were no significant differences at baseline in these volumetric indices in the present study between the diabetic group and the non-diabetic group. In the present study the percent change in EEM volume during the follow-up period indicated a significant negative remodeling in each group. During the remodeling, EEM volume at follow up tended to be larger, and the change in EEM volume tended to be smaller in the diabetic group, although $\mathrm{P}$ value did not reach significance. As already shown in the main JAPANACS study, the change in EEM volume was significantly correlated with that of $\mathrm{PV} .^{8}$ Therefore, this tendency might be considered to be an adaptation process. Non-significant difference in EEM volume at follow up and its change, however, might suggest the existence of a different remodeling mechanism in the 2 groups, because the degree of PV regression was significantly smaller in the diabetic group.

Another important result in the present study was that significant correlations between the percent change in PV and percent change of the LDL-C level or the follow-up LDL-C level could be seen only in the diabetic group, and were absent in the non-diabetic group. Although this correlation coefficient was significant but not so high, this difference between the 2 groups might suggest the existence of both LDL-C-dependent and LDL-C-independent mechanisms of plaque regression induced by statins. Therefore, it can be assumed that some specific or more complex tissue characteristics of plaques in ACS patients with DM, as discussed below, might significantly inhibit the LDL-C-independent mechanism, while plaque regression in ACS patients without DM might be mediated mainly by the LDL-C-independent mechanism. Such switching of the regression mechanism might be determined by the $\mathrm{HbA} \mathrm{Ac}_{\mathrm{c}}$ level, as suggested by the present finding. This suggests that intensive glycemic control might be essential in ACS patients with DM to achieve significant plaque regression. Although the details of the putative LCL-C-independent mechanism are unknown, it might be related to the pleiotropic effects of statin. ${ }^{16-19}$ Whatever the exact mechanism, these data provide the important message that intensive reduction of the LDL-C level should be ensured to obtain a greater degree of regression of the PV in ACS patients with DM.

Williams et al carried out a systematic review of numerous previous in vitro studies, concluding that possible mechanisms responsible for lesion shrinkage include decreased retention of ApoB-lipoproteins within the arterial wall, efflux of cholesterol and other toxic lipids from plaques, emigration of foam cells out of the arterial wall, and influx of healthy phagocytes that remove necrotic debris and other components of the plaque. ${ }^{20}$ It can be assumed that some of these mechanisms are dependent on LDL-C level, and the other ones are independent, which might be attenuated by the presence of DM. But there have been no documented reports so far regarding the relationship between activity of each mechanism and presence of DM, which should be clarified in further studies. Another possible reason for the difference in plaque regression due to statin between DM patients and non-DM patients might be attributed to the difference in the activity of atherosclerotic process to promote plaque formation.

Recently, some studies using new intravascular imaging modalities suggested that the plaque in diabetic patients had a significantly bigger lipid core, ${ }^{21}$ a lesser degree of intimal hyperplasia, ${ }^{21}$ and more abundant dense-calcium or fibrocalcific tissue. ${ }^{22}$ Kawasaki et al demonstrated that the effect of statins on plaque regression was mainly mediated by absorption of the lipid core. ${ }^{6}$ Although no consistent specific tissue characteristics of plaques in diabetic patients have been confirmed, such complex properties of the tissue components of a plaque might prevent the effect of statins of producing regression of the lipid core. Recently, Hirayama et al reported a significant change in plaque color by statin using coronary angioscopy. ${ }^{23}$ Furthermore, a multicenter study has been ongoing to clarify the change in tissue characteristics by statin, using VH-IVUS. ${ }^{24}$ Therefore, further studies particularly focused on the tissue characteristics of plaques may provide further validation of this hypothesis in the near future.

The uniqueness of the regressive mechanism of plaques suggested in diabetic patients could also be observed in relation to other clinical parameters in this group, such as the TC, RLP-C, non-HDL-C and ApoB levels; none of these parameters was correlated with the percent change in PV in the nondiabetic patients. Therefore, concomitant intensive improvement of these parameters by several interventions including therapeutic lifestyle changes or aggressive diet modification would exert favorable effects in ACS patients with DM in terms of the degree of plaque regression. Consequently, the present results have the important clinical implication that ACS patients with DM should be treated with an understanding of the refractory characteristics of plaque regression as well as of the necessity of specific therapeutic interventions.

In addition to the study limitations documented in the main report of the JAPAN-ACS study, ${ }^{8}$ the current substudy had specific limitations. In the present substudy the baseline profile between the 2 groups was not completely matched especially for the proximity of the observed site compared to the PCI site. Although the percent change of the PV was similar between the proximal site and distal site, original difference in the tissue characteristics between the proximal and the distal coronary branch should be considered in the interpretation of the data. ${ }^{25,26}$ The diagnosis of diabetes was made by the attending physician in the participants. Glucose tolerance was not thoroughly examined in some patients. Patients with only impaired glucose tolerance or borderline diabetes were not diagnosed as having DM. Furthermore, no specific interventions to treat $\mathrm{DM}$ were attempted, so that the effects of intensive glucose control on our data could not be elucidated. Therefore, a randomized controlled multicenter study with primary prospective intervention for DM would be needed to confirm our data.

\section{Conclusions}

The presence of DM was one of the major negative determinants of plaque regression induced by statin therapy in patients with ACS. There were significant correlations, however, between the percent change in PV and percent change 
of the LDL-C level or follow-up LDL-C level only in patients with DM, while no such significant correlations were found in the patients without DM. Therefore, these data suggest the importance of more intensive reduction of the LDL-C levels to achieve a greater degree of regression of the PV in ACS patients with DM.

\section{Acknowledgments}

We sincerely acknowledge the contributions of Izumi Miki, Saeko Minematsu, Yumika Fujino and Miya Hanazawa to the data management, and those of Hiroko Kanou, Natsuko Yamamoto, Tatsuhiro Fujimura and Genta Hashimoto to the IVUS core-laboratory management and IVUS planimetry. JAPAN-ACS Investigators: Aichi Medical University (Takayuki Ito, MD; Hiroaki Takashima, MD); Akita Medical Center (Tadaya Sato, MD); Anjo Kosei Hospital (Masato Watarai, MD); Ehime Prefectural Central Hospital (Yukio Kazatani, MD; Shinichi Hiramatsu, MD); Fujita Health University (Hitoshi Hishida, MD; Yukio Ozaki, MD; Hiroyuki Naruse, MD); Fukuoka University (Keijiro Saku, MD; Shin-ichiro Miura, MD; Atsushi Iwata, MD); Gifu Prefectural General Medical Center (Sachiro Watanabe, MD; Toshiyuki Noda, MD, Ryuhei Tanaka, MD; Koji Ono, MD); Hiroshima City Asa Hospital (Keigo Dote, MD; Masaya Kato, MD; Shota Sasaki, MD; Kentaro Ueda, MD); Hiroshima City Hospital (Ichiro Inoue, MD; Takuji Kawagoe, MD); Japanese Redcross Nagoya First Hospital (Haruo Kamiya, MD); Juntendo University (Hiroyuki Daida, MD; Katsumi Miyauchi, MD); Juntendo University Shizuoka Hospital (Satoru Suwa, MD); Juntendo University Urayasu Hospital (Yuji Nakazato, MD; Kosei Tanimoto, MD); Kansai Rosai Hospital (Shinsuke Nanto, MD; Fusako Sera, MD); Kokura Memorial Hospital (Masakiyo Nobuyoshi, MD; Hitoshi Yasumoto, MD); Kurashiki Central Hospital (Kazuaki Mitsudo, MD; Yasushi Fuku, MD); Kyoto University (Takeshi Kimura, MD; Yoshihisa Nakagawa, MD); National Cardiovascular Center (Hiroshi Nonogi, MD; Yoritaka Otsuka, MD; Nobuaki Kokubu, MD); National Hospital Organization Hamada Medical Center (Hiroshi Iida, MD; Mutsuo Nakayama, MD); National Hospital Organization Kagoshima Medical Center (Shinichi Minagoe, MD; Hitoshi Nakajima, MD; Masahiro Sonoda, MD); NTT East Corporation Sapporo Hospital (Tetsuro Kouya, MD; Noriyuki Miyamoto, MD; Yoshiyuki Suzuki, MD; Sachie Taguchi, MD); Okayama Redcross General Hospital (Toru Ujihira, MD; Hironori Saitou, MD; Tetsuya Sato, MD); Osaka City General Hospital (Akira Itoh, MD; Kei Yunoki, MD); Saiseikai Kumamoto Hospital (Takashi Honda, MD; Koichi Nakao, MD; Norihiro Yamada, MD); Sendai Kosei Hospital (Taiichiro Meguro, MD; Kaname Takizawa, MD); Tokuyama Central Hospital (Hiroshi Ogawa, MD; Takahiro Iwami, MD); Toranomon Hospital (Sugao Ishiwata, MD; Yo Fujimoto, MD); Tsuchiya General Hospital (Yasuhiko Hayashi, MD; Mamoru Toyofuku, MD; Masaya Otsuka, MD); University of Tokyo (Yasunobu Hirata, MD; Jiro Ando, MD); Wakayama Medical University (Takashi Akasaka, MD; Takashi Kubo, MD; Hironori Kitabata, MD); Yamaguchi University (Masunori Matsuzaki, MD; Takafumi Hiro, MD; Takashi Fujii, MD; Takayuki Okamura, MD; Jutaro Yamada, MD; Genta Hashimoto, MD; Tatsuhiro Fujimura, MD); Yokkaichi Municipal Hospital (Satoshi Ichimiya, MD; Masaaki Kanashiro, MD; Junji Watanabe, MD; Seiji Fukui, MD; Taiki Ohashi, MD; Nobutake Kurebayashi, MD); Yokohama City University Medical Center (Kiyoshi Hibi, MD).

\section{References}

1. Schwartz GG, Olsson AG, Ezekowitz MD, Ganz P, Oliver MF, Waters D, et al. Effects of atorvastatin on early recurrent ischemic events in acute coronary syndromes: The MIRACL study: A randomized controlled trial. JAMA 2001; 285: 1711-1718.

2. Cannon CP, Braunwald E, McCabe CH, Rader DJ, Rouleau JL, Belder $\mathrm{R}$, et al. Intensive versus moderate lipid lowering with statins after acute coronary syndromes. N Engl J Med 2004; 350: 1495-1504.

3. Thompson PL. Clinical relevance of statins: Instituting treatment early in acute coronary syndrome patients. Atheroscler Suppl 2001; 2: $15-19$.

4. Nissen SE, Tuzcu EM, Schoenhagen P, Brown BG, Ganz P, Vogel RA, et al. Effect of intensive compared with moderate lipid-lowering therapy on progression of coronary atherosclerosis: A randomized controlled trial. JAMA 2004; 291: 1071-1080.

5. Nissen SE, Nicholls SJ, Sipahi I, Libby P, Raichlen JS, Ballantyne $\mathrm{CM}$, et al. Effect of very high-intensity statin therapy on regression of coronary atherosclerosis: The ASTEROID trial. JAMA 2006; 295: $1556-1565$.

6. Kawasaki M, Sano K, Okubo M, Yokoyama H, Ito Y, Murata I, et al. Volumetric quantitative analysis of tissue characteristics of coronary plaques after statin therapy using three-dimensional integrated backscatter intravascular ultrasound. J Am Coll Cardiol 2005; 45: 1946- 1953.

7. Okazaki S, Yokoyama T, Miyauchi K, Shimada K, Kurata T, Sato $\mathrm{H}$, et al. Early statin treatment in patients with acute coronary syndrome: Demonstration of the beneficial effect on atherosclerotic lesions by serial volumetric intravascular ultrasound analysis during half a year after coronary event: The ESTABLISH Study. Circulation 2004; 110: $1061-1068$.

8. Hiro T, Kimura T, Morimoto T, Miyauchi K, Nakagawa Y, Yamagishi M, et al. Effect of intensive statin therapy on regression of coronary atherosclerosis in patients with acute coronary syndrome: A multi-center randomized trial evaluated by volumetric intravascular ultrasound using pitavastatin versus atorvastatin (JAPAN-ACS study). J Am Coll Cardiol 2009; 293: 293-302.

9. Nicholls SJ, Tuzcu EM, Crowe T, Sipahi I, Schoenhagen P, Kapadia $\mathrm{S}$, et al. Relationship between cardiovascular risk factors and atherosclerotic disease burden measured by intravascular ultrasound. J Am Coll Cardiol 2006; 47: 1967-1975.

10. Sipahi I, Tuzcu EM, Schoenhagen P, Wolski KE, Nicholls SJ, Balog $\mathrm{C}$, et al. Effects of normal, pre-hypertensive, and hypertensive blood pressure levels on progression of coronary atherosclerosis. $J \mathrm{Am}$ Coll Cardiol 2006; 48: $833-838$.

11. Nicholls SJ, Tuzcu EM, Sipahi I, Schoenhagen P, Hazen SL, Ntanios F, et al. Effects of obesity on lipid-lowering, anti-inflammatory, and antiatherosclerotic benefits of atorvastatin or pravastatin in patients with coronary artery disease (from the REVERSAL Study). Am J Cardiol 2006; 97: 1553-1557.

12. Sipahi I, Tuzcu EM, Wolski KE, Nicholls SJ, Schoenhagen P, Hu $\mathrm{B}$, et al. Beta-blockers and progression of coronary atherosclerosis: Pooled analysis of 4 intravascular ultrasonography trials. Ann Intern Med 2007; 147: 10-18

13. Miyauchi K, Kimura T, Morimoto T, Nakagawa Y, Yamagishi M, Ozaki Y, et al. Japan assessment of pitavastatin and atorvastatin in acute coronary syndrome (JAPAN-ACS): Rationale and design. Circ J 2006; 70: 1624-1628.

14. Donahoe SM, Stewart GC, McCabe CH, Mohanavelu S, Murphy SA, Cannon CP, et al. Diabetes and mortality following acute coronary syndromes. JAMA 2007; 298: 765-775.

15. Nicholls SJ, Tuzcu EM, Kalidindi S, Wolski K, Moon KW, Sipahi $\mathrm{I}$, et al. Effect of diabetes on progression of coronary atherosclerosis and arterial remodeling: A pooled analysis of 5 intravascular ultrasound trials. J Am Coll Cardiol 2008; 52: 255-262.

16. Masamura K, Oida K, Kanehara H, Suzuki J, Horie S, Ishii H, et al. Pitavastatin-induced thrombomodulin expression by endothelial cells acts via inhibition of small $\mathrm{G}$ proteins of the Rho family. Arterioscler Thromb Vasc Biol 2003; 23: 512-517.

17. Bonnet J, McPherson R, Tedgui A, Simoneau D, Nozza A, Martineau $\mathrm{P}$, et al. Comparative effects of $10-\mathrm{mg}$ versus $80-\mathrm{mg}$ atorvastatin on high-sensitivity C-reactive protein in patients with stable coronary artery disease: Results of the CAP (Comparative Atorvastatin Pleiotropic effects) study. Clin Ther 2008; 30: 2298-2313.

18. Nakamura K, Sasaki T, Cheng XW, Iguchi A, Sato K, Kuzuya M. Statin prevents plaque disruption in apoE-knockout mouse model through pleiotropic effect on acute inflammation. Atherosclerosis 2009; 206: 355-361.

19. Liu PY, Liu YW, Lin LJ, Chen JH, Liao JK. Evidence for statin pleiotropy in humans: Differential effects of statins and ezetimibe on rho-associated coiled-coil containing protein kinase activity, endothelial function, and inflammation. Circulation 2009; 119: $131-138$.

20. Williams KJ, Feig JE, Fisher EA. Rapid regression of atherosclerosis: Insights from the clinical and experimental literature. Nat Clin Pract Cardiovasc Med 2008; 5: 91-102.

21. Sano K, Kawasaki M, Okubo M, Yokoyama H, Ito Y, Murata I, et al. In vivo quantitative tissue characterization of angiographically normal coronary lesions and the relation with risk factors: A study using integrated backscatter intravascular ultrasound. Circ J 2005; 69: $543-549$.

22. Nasu K, Tsuchikane E, Katoh O, Fujita H, Surmely JF, Ehara M, et al. Plaque characterisation by virtual histology intravascular ultrasound analysis in patients with type 2 diabetes. Heart 2008; 94: $429-433$

23. Hirayama A, Saito S, Ueda Y, Takayama T, Honye J, Komatsu S, et al. Qualitative and quantitative changes in coronary plaque associated with atorvastatin therapy. Circ J 2009; 73: 718-725.

24. Nozue T, Yamamoto S, Tohyama S, Umezawa S, Kunishima T, Sato A, et al. Treatment with statin on atheroma regression evaluated by intravascular ultrasound with virtual histology (TRUTH Study): Rationale and design. Circ J 2009; 73: 352-355.

25. Tanaka A, Imanishi T, Kitabata H, Kubo T, Takarada S, Kataiwa 
$\mathrm{H}$, et al. Distribution and frequency of thin-capped fibroatheromas and ruptured plaques in the entire culprit coronary artery in patients with acute coronary syndrome as determined by optical coherence tomography. Am J Cardiol 2008; 102: 975-979.

26. Komura N, Hibi K, Kusama I, Otsuka F, Mitsuhashi T, Endo M, et al. Plaque location in the left anterior descending coronary artery and tissue characteristics in angina pectoris: An integrated backscatter intravascular ultrasound study. Circ J 2010; 74: 142-147.

\section{Appendix 1}

Steering committee: Takafumi Hiro, MD; Katsumi Miyauchi, MD; Yoshihisa Nakagawa, MD; Masakazu Yamagishi, MD; Yukio Ozaki, MD (working members, respectively); Takeshi Kimura, MD; Hiroyuki Daida, MD (co-principal investigators, respectively); Masunori Matsuzaki, MD (principal investigator). Independent event assessment committee: Kazuo Kimura, MD; Satoshi Saito, MD; Tetsu Yamaguchi, MD. Independent statistician: Takeshi Morimoto, MD.

\section{Appendix 2}

Baseline Parameters

The following 83 variables at baseline were considered as factors poten- tially associated with the \% change of the coronary PV and entered into the multivariate analysis model: baseline characteristics (age, gender, diabetes, medication for diabetes, insulin treatment for diabetes, history of MI, history of angina, history of PCI, history of CABG, history of blood vessel diseases, history of DAA, history of aortic aneurysm, history of ASO, history of CI, hypertension, family history of CAD, smoking, alcohol drinker, drug allergy, height, body weight, BMI, waist circumference, SBP, DBP, heart rate, type of ACS, stent usage, type of stent, culprit vessel, proximal/distal to the treated site, abnormal Q wave positive, max CPK, troponin $\mathrm{T}$ positive, troponin I positive); concomitant drugs (Ca blocker, nitrate, ACE inhibitor, ARB, $\beta$-blocker, diuretic, aldosterone blocker, digitalis, PPAR- $\gamma$ agonist, sulfonyl urea, $\alpha$-GI, ticlopidine, clopidogrel, aspirin, other antiplatelet agents, warfarin, anti arrhythmic agent); baseline blood parameters (TC, LDL-C, TG, HDL-C, HDL2-C, HDL3-C, RLP-C, small dense LDL, non-HDL-C, LDL-C/HDL-C ratio, ApoA-I, ApoB, ApoE, ApoB/ApoA-I ratio, MDA-LDL, phospholipid, Lp(a), hs-CRP, PTX3, HbAlc); baseline IVUS parameters (lesion length of evaluation site, $\mathrm{PV}, \% \mathrm{PV}$, normalized PV, vessel volume, minimum lumen area in evaluation site, lumen volume, minimum lumen area in treated site, plaque area in treated site, vessel area in treated site, stent area in treated site). 